\title{
Proceeding
}

10th INSHS International Christmas Sport Scientific Conference, 4-5 December 2015. International Network of Sport and Health

Science. Szombathely, Hungary

\section{Development of an analysis of swimming techniques using instrumentation and the development of a new measurement method at Brno University of Technology}

\author{
BÁTOROVÁ MICHAELA ${ }^{1}$, JAN ŠŤASTNÝ ${ }^{1}$, MOTYČKA JAROSLAV ${ }^{1}$, JANURA MIROSLAV ${ }^{2}$ \\ 1 Centre of Sport Activities, Brno University of Technology, Brno, Czech Republic \\ 2 Faculty of Physical Culture, Palacký University, Olomouc, Czech Republic
}

\begin{abstract}
This article provides an overview of the development of measuring methods and instrumentation for the analysis of swimming techniques around the world, and describes in more detail the Tachograph method used in BUT (Brno University of Technology), including information about the currently developed method for measuring the swimmers using accelerometers. Our aim is to present a comprehensive view of the issue and describe the direction of the current development of measurement techniques used in swimming. The article further describes in detail the physical nature of the measurement methods and discusses their pros and cons. In addition to conventional methods (speedometer, accelerometer), we also mention unconventional methods (CFD method, 3D motion analysis etc.). The main contribution of the paper is in highlighting new directions and methods of measurement in swimming, including a specific approach for the evaluation of swimming techniques at the Brno University of Technology (BUT), where the scientific team at the Center of Sports Activities (CESA) has for several years developed and refined a tachograph measuring method with synchronous underwater video recording of swimmers. This method was used with swimmers from the Czech national swimming team and from Czech sports secondary schools as part of ongoing research and efforts towards more accurate measurements. Finally, the article describes current developments in measuring methods done at BUT using accelerometers. Key words: ANALYSIS OF SWIMMING, SPEEDOMETER, ACCELEROMETER, TACHOGRAPH, METHODS OF MEASURING.
\end{abstract}

Corresponding author. Centre of Sport Activities, Brno University of Technology, Technická 2896/2, 61669 Brno, Czech Republic

E-mail: batorova@cesa.vutbr.cz

10th INSHS International Christmas Sport Scientific Conference, 4-5 December 2015. International Network of Sport and

Health Science. Szombathely, Hungary.

JOURNAL OF HUMAN SPORT \& EXERCISE ISSN 1988-5202

(c) Faculty of Education. University of Alicante

doi:10.14198/jhse.2016.11.Proc1.05

VOLUME 11 | Proc1 | 2016 | S146 


\section{INTRODUCTION}

The global competitive swimming is still pushing the boundaries of swimming performance closer to physiological capabilities of swimmers. Therefore there is a growing need to incorporate instrumental measurement into the swimmers training process with analysis to eliminate mistakes in swimming technique that could limit the swimmers in achieving better times, or could in the long run lead to damage to the musculoskeletal system. Prior to the forthcoming Olympic Games in Rio de Janeiro, requirements for the best possible preparation of swimmers increases, and there is a growing interest from both coaches and swimmers in methods for the analysis of swimming techniques.

In the first part of the paper, we will focus on Computational Fluid Dynamics (CFD), allowing evaluation of the hydrodynamic forces acting on the swimmers hands. Then we will take a look at the 3D motion analysis in aquatic environments using camera systems and specialized software. Further, we will focus on conventional measurement methods such as Swim speedo-meter, which is commercially available and works on a similar principle as the Tachograph from Professor Motyčka, which we use in our research. Other conventional methods discussed here include measurements with accelerometers. We will look in more detail at the prototype of Ciren measuring device and its comparison with the speedo meter and video method. In the last part of the article, we will describe the Tachograph method of swimmer measuring, which we use at BUT for measuring the Czech national swimming team and students of sports secondary schools, including a description of the current state of development of new a measurement method using accelerometers and software designed for this purpose, including an outline of the future direction of development, in which we would like to continue aiming our research.

\section{New unconventional methods of swimmers measurements}

Computational Fluid Dynamics (CFD)

Presenting the measuring methods, which are used worldwide to measure swimmers, we can start with a method that works on the basis of simulations of the swimming stroke and examines hydrodynamic effect of the aquatic environment.

Sato and Gino (2013) form Turkey describe a system that was developed for the analysis of the stroke based on CFD (Computational Fluid Dynamics), which allows to evaluate the hydrodynamic forces acting on the swimmers hands. The developed system of analysis takes into account the effect of the swimmer's hand stroke at a certain time point, including the acceleration and to a certain extent the curvature of the stroke trajectory. It is also necessary to solve the Navier-Stokes equation with numerical method, which is used to solve CFD calculation for viscous fluid flow around the swimmer's hand, and this method also takes account of the complicated hand geometry. CFD calculation method is verified by comparison with experiments at steady state or with changing parameters (transients). After validation, analysis of the swimmer's stroke was designed, which reflects the stroke trajectory measured with synchronized cameras and the liquid pressure acting from sides was calculated using CFD. As a demonstration of stroke analysis, two world class swimmers were selected in $200 \mathrm{~m}$ freestyle race. Hydrodynamic forces acting on the hands of the top swimmers were counted and compared for two swimmers. The faster swimmer, whose stroke shifted him to the speed of $1.84 \mathrm{~m} / \mathrm{s}$, generated more power at higher efficiency than the slower swimmer, who moved up to $1.75 \mathrm{~m} / \mathrm{s}$. The applicability of this method of analysis of the swimmers stroke was demonstrated using the published analysis. This method seems to be very complicated and instrumentally challenging for inclusion in the coaching practice; however, it is certainly beneficial for theoretical contribution and expansion of knowledge in the field of swimming biomechanics. 
Analysis of underwater motion using a 3D system

Another method for analyzing the swimmers underwater motion are 3D measurement systems that use cameras and specially developed software.

The University of Bologna conducts research into 3D analysis of human motion. Magalhaes and his colleagues Sawaeha, Rocco, Cortesi, Gatta and Fantozzi (2013) in their book compare two kinds of software concerning their usefulness for analysis of human motion in the aquatic environment. They focused not only on swimming but also on underwater walking, including walking on an underwater treadmill. They used 6 underwater cameras and reflective points placed on the body of test persons for the 3D analysis. The authors themselves admit that the rapid swimming locomotion causes water swirling and creates bubbles, which greatly distorts the visibility of reflective points that make it possible to eventually render the model of swimmers motion. The DVP software in the study showed to be more suitable for the analysis of human motion due to greater degree of automation and less need for intervention in the measurement other than COM software (OTC).

Scientists at the University of Porto also developed a method for the analysis of swimming techniques using a set of cameras. The method is based on computer image processing from 4 underwater cameras and two cameras above the water surface.

For the reconstruction of 3D scenes from two or more images, epipolar geometry is used, which is based on projective linear transformation. This transformation is also called a homography and expresses the changing perception of an object (in this case swimmer) when changing the position and angle of observation. Basically the result is then a computerized motion of the swimmers or their limbs, as shown by comparing pixels from each camera image.

The problem of this method lies in the difficult processing of images from underwater cameras due to the aquatic environment and air / water interface. Changing light conditions can also result in complications. This analysis is suitable for indoor pools, but for outdoor pools, the possibility of processing images from the cameras is very limited. A big advantage is no restriction of the swimmer and, in the case of permanently installed cameras in the pool environment, no need of technical preparations for measurement.

As discussed herein, at present, the development of research in swimming starts heading for the analysis of swimming techniques using a computerized video processing. These methods are based on image processing by epipolar geometry, which allows the computer vision and detection of specific points (e.g. reflective - Simi Motion, see Fig. 1) in the image. The software processing of the image provides the necessary indicators and parameters for qualitative assessment of swimming techniques. It is very likely that the development of these methods will be crucial for the analysis of swimming techniques in the future. 


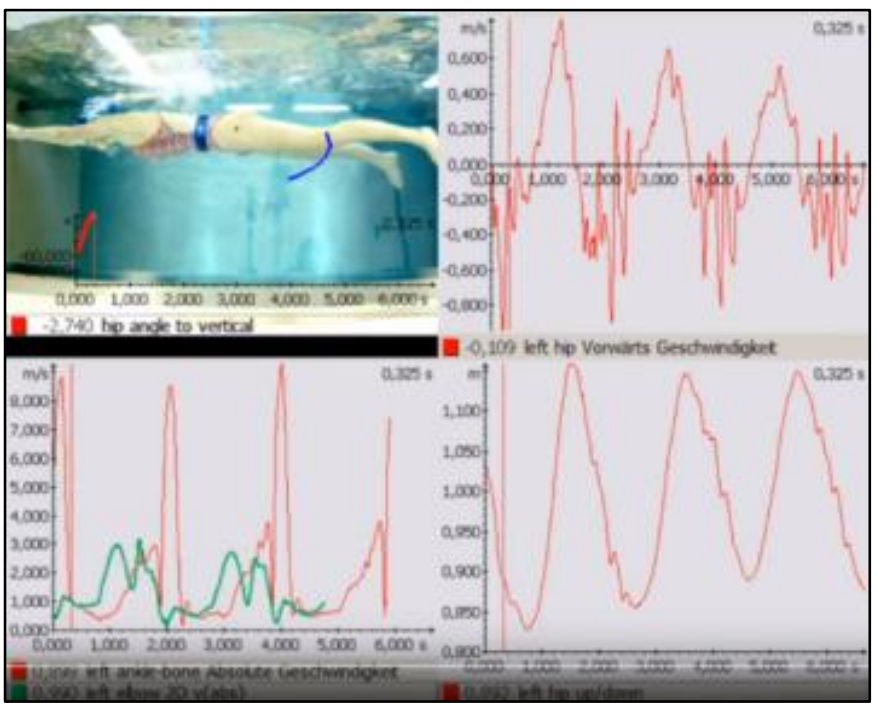

Figure 1. Analysis of swimmers motion with Simi Motion (Simi, 2015)

\section{Conventional methods of measuring in the world}

Speedometer

Intracyclic swim stroke velocity meter (speedometer) from Swim Sportec is a commercially available device used to measure the speed of swimming and to analyze swimming technique from the graphical display of the instantaneous speed. The equipment usually includes a measuring card (an interface between dynamo voltage and computer signal) and software application that lets you view and process the measured data online and on record. This device operates on the basis of unwinding a cable that is pulled by the swimmer and the software graphically records the speed course from unwinding speed and drops in rpm. Authors of the research article set out to verify the validity of the method of measuring the speed in a horizontal axis using a speedo-meter and videometry (processing images from the cameras with a sampling frequency of $50 \mathrm{~Hz}$ ). Three validation criteria were gradually investigated: a) Paired t-test (validation criterion $a \geq 0.05$ ); b) linear regression model (validation criterion $\mathrm{R}^{2} \geq 0.49$ ); c) Blant-Altman graph (validation criterion: at least $80 \%$ of the chart within the deviation \pm 1.96 ). All three validation criteria were met, so the speedometry method proved to be useful for measuring the horizontal speed of swimming (Barbosa, Costa, Morais, Jesus, Silva, \& Batista Goncalves, 2011).

New approach in processing data from an inertial sensor

Possibility of speed measurement in aquatic environment without any limitation to swimmers is a major challenge for engineers and scientists in the field of sport. In the past, video systems were the first choice in monitoring and analysis of swimmers during training. Application of small inertial sensors allowed the emergence of new methods for swimmer's motion analysis. These small systems are lightweight, easy to use and do not restrict swimmers during training, allowing easy monitoring, which after analysis leads to improvements in training.

The research of authors from Hong Kong (Stamm, Burkett, Hagema \& Theil, 2013) described in this publication, uses their own developed low-cost system of inertial sensors, which are attached to the lower back and allow to record acceleration of swimmers. The recorded data were processed in MATLAB. Measurements were performed and analyzed for seven swimmers. Measured speed was compared with the attached cable accelerometer and analyzed by regression and Bland-Altman speed analysis. Measurements 
were performed for the three levels of swimmers effort (maximum speed, average-mean and slow pace). In their publication, the authors describe a completely new approach to data processing from accelerometers. It is also important that the use of accelerometer based measurements for analysis of swimming techniques is currently limited because of the accuracy. However, this issue is slowly fading thanks to gradual development of innovative technologies.

Measurements of swimmers using the Ciren measuring device prototype, tachometer (speedometer) and a multi-camera system (3D video)

The Ciren prototype is based on NANO MEMSense inertial measurement unit, which consists of a combined three-axis accelerometer $( \pm 5 \mathrm{~g})$, a magnetometer ( \pm 1.9 Gauss) with a gyroscope $( \pm 600 \% \mathrm{~s})$ and an integrated control unit which records data with a frequency of $150 \mathrm{~Hz}$. The measuring system is inserted into the sealed casing (IP68, external dimensions are 117 × 80 × 33 mm) (Puel, Seifert, \& Hellard, 2014).

This prototype was tested in several tests. The first one was conducted in a mechanical laboratory where Ciren was mounted on a Mikron HSM 600U, a five-axis machining center, which allows to program and control chosen trajectories. Various sets of movements where tested (50 repetitions).

The other two experiments were then performed in a pool on a group of experienced swimmers. Participants wore the Ciren accelerometer on their lower back, mounted with a belt and loops specially designed for the device so that it can be connected securely (Figure 2).

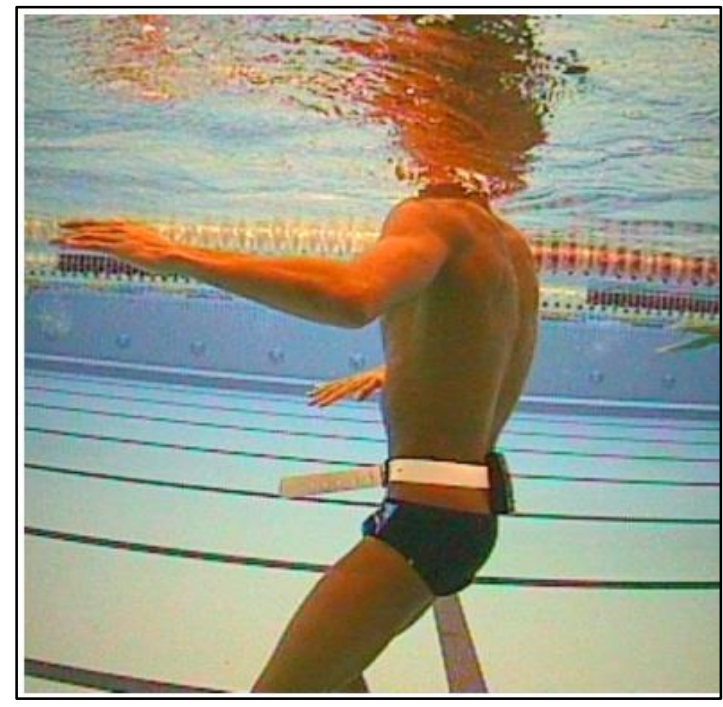

Figure 2. Test person with a mounted accelerometer 


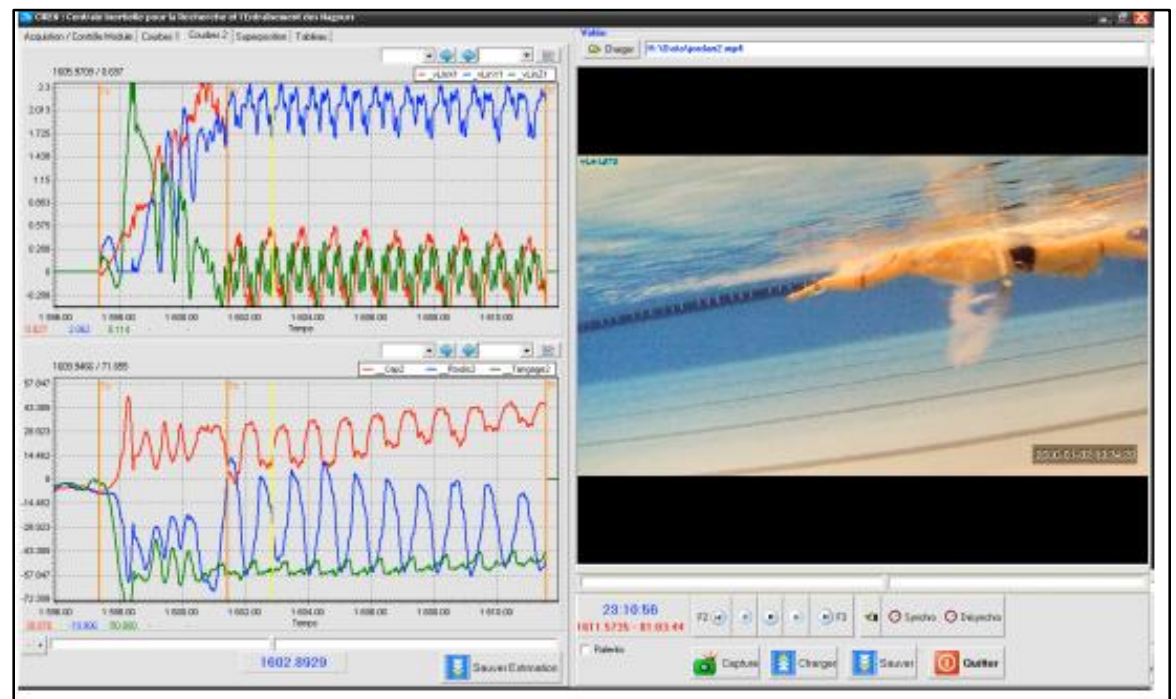

Figure 3. Measurement and evaluation of the swimmer's speed using Ciren v6.0 software

The longitudinal speed of swimmers was measured and verified by three methods: using Ciren v6.0 software (Fig. 3), which integrates all embedded sensors and pre-defined initial conditions (starting position of swimmers and length of the pool), tachometer (speedometer) and a multi-camera system (3D video).

In comparison with a mechanical system of measurement, an accelerometer (Ciren) measures the rotational speed and the translational acceleration, which have been verified on the basis of test range of motion.

The accelerometer (Ciren) calculates the longitudinal speed of the swimmer from the measured data; and in comparison with a speedometer, we get a lower amplitude, probably due to the construction of speedometer (motor used as a generator, which measures the speed of the wire unwinding), and also because of the moment of inertia in the mechanical measurement.

In addition, the average speed from the speedometer appears to be lower than the average speed calculated by accelerometer (Ciren). Minor inaccuracies in the measurement of average speed for the accelerometer (Ciren), caused by a drift, were evident in the practical test, however, the speed curve and frequency of the swimmer's strokes were not affected. The third experiment showed good agreement between measurements with the accelerometer (Ciren) and 3D kinematic analysis. Longitudinal velocity calculated by the Ciren device (accelerometer) can be considered accurate in the context of analysis of swimming techniques. The measured speed curve clearly shows the cycles of strokes and allows to easily control the strokes frequencies.

Accelerometer (Ciren), offers the possibility to measure the instantaneous speed of swimmers and also allows to analyze phases of strokes and the frequency. Compared with conventional systems, it allows continuous measurement over the entire length of the pool, even at longer measurements than one length of the pool.

In addition to the fact that the use of measured data from the accelerometer (Ciren) with a video record allows accurate analysis of swimming techniques, it also facilitates the measurement (no complicated setup), minimizes any restrictions to the swimmer, and finally, accelerates feedback to the operator (coach, sports scientist, etc.). 
The authors suggest that in the future, it would be interesting to compare accelerometer prototype and other mechanical systems in the laboratory, e.g. their ability to reproduce 3D motion of a swimmer with a converter to verify the accuracy of the Ciren accelerometer for the evaluation of speed (Puel, Seifert, \& Hellard, 2014).

We discussed this method in detail particularly because our research at Brno University of Technology, Centre of Sports Activities, is routed to similar research direction in which (after the completion of the development of measurement systems using accelerometers with synchronous video recording) we would like to perform comparison measurements with the Tachograph device from prof. Motyčka along with the camera system with which we currently measure and analyze swimming techniques of Czech representative swimmers and swimmers from sports secondary schools throughout the Czech Republic.

\section{Research at Brno University of Technology}

Tachograph measuring method

Prof. Motyčka started studying instantaneous speed and acceleration in swimming in the seventies of the 20th century; he also initiated the construction of the Tachograph measuring device and conducted extensive measurements of Czechoslovak elite swimmers with this device in 1971-1974. A similar method, where the swimmer is wired with the measuring tool on the bank, was used by Miyshita in 1975 (Motyčka, 2001).

Research and development using the tachograph measurement method

Motyčka (2001) states that Tachograph recorded uncoiled length, speed and acceleration, and these values were recorded by multi-channel recorder, later by measuring tape recorder and multilevel counter. Average speed, speed range and mechanical efficiency were evaluated. Since in the early stages of tachograph method development, measured values were recorded on graph paper and then read, there was an error in the measurement and distortion in measured values. Further inaccuracy was caused by low number of measured values in a given time interval, causing a distortion of recorded curve. The measuring device was mounted on one side of the pool for the coil with tachodynamo to be at a height of $60 \mathrm{~cm}$ above the water surface and the device measured only in the direction away from it. In the nineties, innovations of measurement methods were made, and as described by Lukášek, data were transferred to portable computers using analog-to-digital converter, and stored in the form of coordinates. Data could be recorded using an AD converter and the Nextwiev NV Um4 program. The measured data were recorded in the form of coordinates and stored on a floppy disk without time coordinates. These values were reformatted from text to numeric form in Excel 97 and were then mathematically processed. The device recorded 200 records per second for 10 seconds. During that time, the swimmer performed 6-8 swimming cycles, and every third or fourth cycle was subjected to analysis. Due to the high sensitivity of the device $(0,0001 \mathrm{~V})$, the curve was not smooth and was therefore adjusted using a mathematical method of moving average (12 values) so as to preserve the extreme displacements of speed and also suppress unimportant and unwanted variations in the graphical plot of the measured values on the curve (Lukášek, 2000).

In the years 2011 - 2015, the tachograph method went through extensive innovations. The construction of tachograph changed and a system of two tachographs evolved (see Fig. 4), which measures the swimmers there and back. The tachograph was software-synchronized and software development and expansion of its additional features and functions are still in progress. Original cameras were replaced with HD quality cameras, and several trolley prototypes were constructed, used by the operator to carry a camera, which allows the side view recording of the swimmers throughout their swimming. At present, in the evaluation of swimming techniques, we use synchronous video from two static cameras (front and back) and a side camera that is mounted on the trolley together with lighting to illuminate swimmers in the pool under poor light conditions. 
During the measurement, the swimmer is now connected to the tachograph using a waist belt made of rigid PVC fabrics tailored for the needs of measurement. The swimmer uses a carabiner to clip on a braided flax rope, which replaced the thin steel cable that used to tangle during swimmers turns and restricted the swimmers. Belt loops to attach the swimmer to the tachograph are located in the middle of a belt, which is attached to the pelvic bones to prevent its movement. Via the flax rope, the swimmer pulls a moving rider mounted on a steel wire pointing from one tachograph to another across the pool. The test person moves forward, dragging the rider while and winding the flax rope, while tachographs are recording speed data and cameras are capturing video of the underwater swimmer. SwimDataViewer Sofware displays graphic speed and acceleration time course from the measured data along with synchronized video record from three underwater video cameras (see Fig. 5).

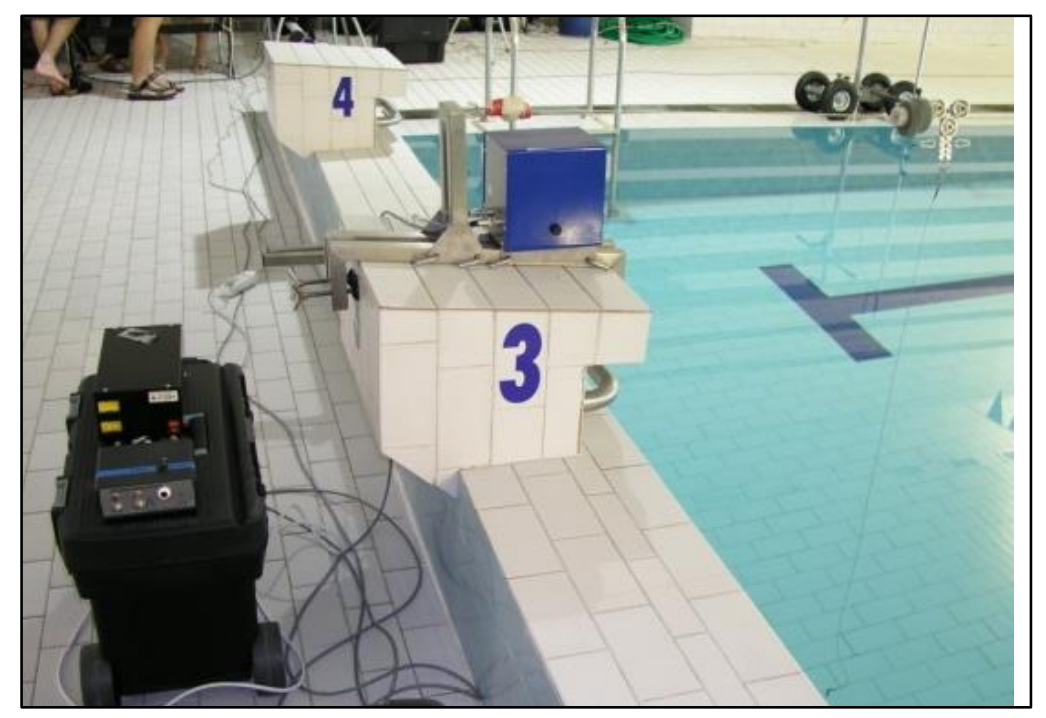

Figure 4. One of the tachographs

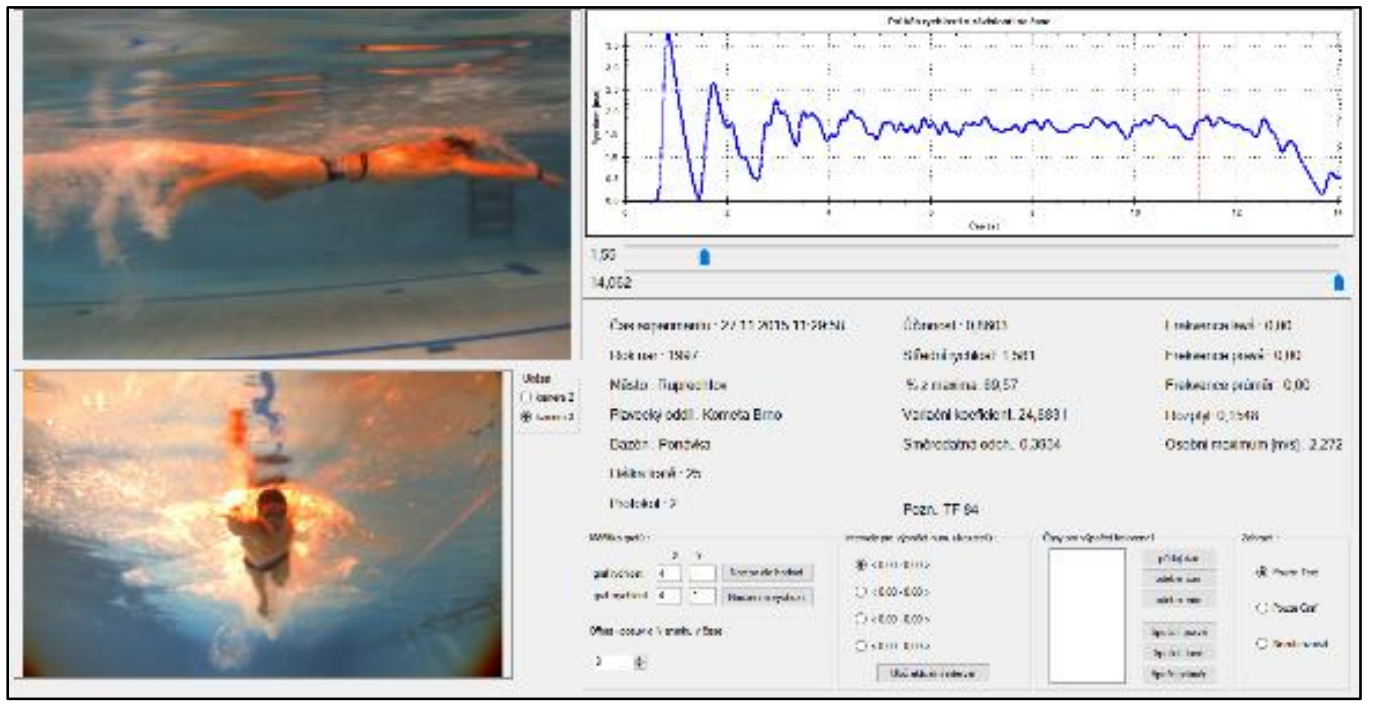

Figure 5. SwimDataViewer Sofware

From the instantaneous speed, the software further shows a calculated graph of acceleration, and calculates the average speed, variance, coefficient of variation, standard deviation, number of swimmer's strokes and 
efficiency of swimming technique which is taken as work performed at a constant speed. Efficiency (\%) of swimming technique according to Motyčka is calculated from work done while swimming. From the physical point of view, it is a ratio of work performed at a constant speed to actual work performed at fluctuating speed. For the best efficiency and thus the effectiveness of swimming, the swimmer should learn to swim at the highest possible speed and with the lowest possible fluctuation. Each measured segment should ideally reach the efficiency of the technique as close as possible to $100 \%$ at the same time maintaining the highest possible speed the swimmer is able to reach (Motyčka, Štastný, Lepková, Pašek \& Bátorová, 2013).

$$
\text { Efficiency calculation: } \quad \eta=\frac{\text { work at constant speed }(\text { lower })}{\text { wort at actual speed }(\text { higher })}=\frac{k \bar{v}^{3} t}{k \Delta t \sum_{i=1}^{n} v_{i}^{3}}
$$

At present, we use this measurement method to measure top racing swimmers and using swimming techniques analysis, video and graphical display of speed, number of strokes, and other values, we analyze mistakes in swimming technique of Czech national swimming team and of swimmers from sports secondary schools and we pass this information to coaches in practice. Currently, our team is working on a research of specific elements of swimming techniques, we analyze and evaluate them, and we prepare the Czech national swimming team for the Summer Olympics in Rio de Janeiro. At the same time, we plan to further develop this method and optionally synchronize with accelerometry method.

\section{Development of a new measurement method using accelerometers}

At the Technical University in Brno, we started the development of a new measurement system using accelerometers in 2015. Our team is working to develop the hardware part, several prototypes were constructed, including a weatherproof case. Our colleagues in the Faculty of Mechanical Engineering, Department of Mechatronics and Robotics, with whom we collaborate, design and build this hardware device so as to be able to record the acceleration of swimmers. Developed software then processes the measured acceleration values to obtain a graphic record of speed. Our team also works on overall waterproof encapsulation, mounting on the body of a swimmer and a reduction of the device size to prevent unwanted interference with the swimming technique, or discomfort while swimming with the device (see Fig. 6).

In the beginning, we tried online data transmission, but the aquatic environment is not suitable for signal transmission and the signal was often interrupted, so we refrained from the online transmission idea. At the moment, the device works on the principle of storing data directly into the measuring device, and when the test person is done with swimming, measured data are wirelessly transferred to PC and can be further processed. In the early stages of testing the measuring device using three-axis accelerometers, we displayed graphically all the axes (see Fig. 7). Now, our development team was able to show the axes separately (Fig. 8) and we are working on software processing of acceleration, which would allow graphic display of acceleration and speed course and will broaden the possibilities of views, zoom and other user functions needed for detailed evaluation of swimming techniques. 


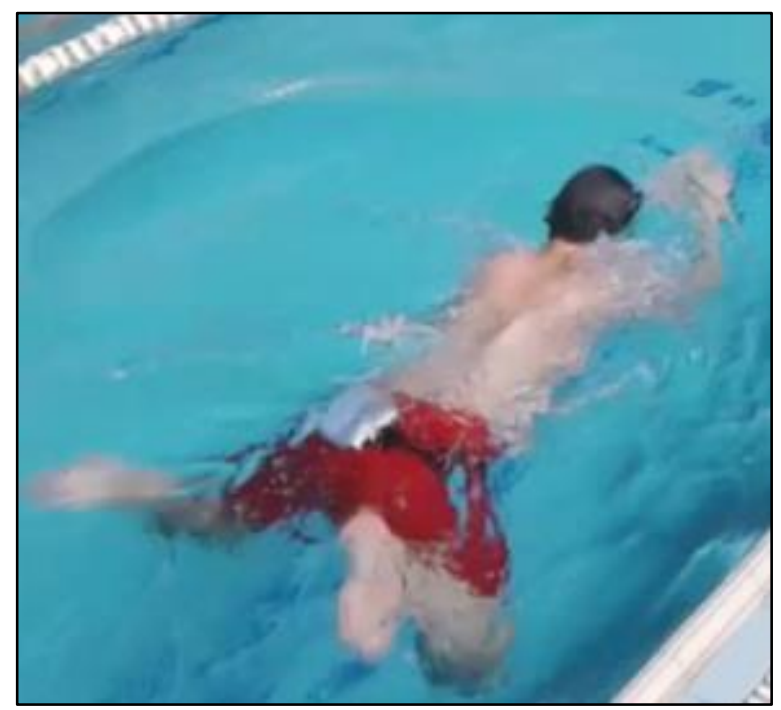

Figure 6. Test swimmer with the first prototype of the device

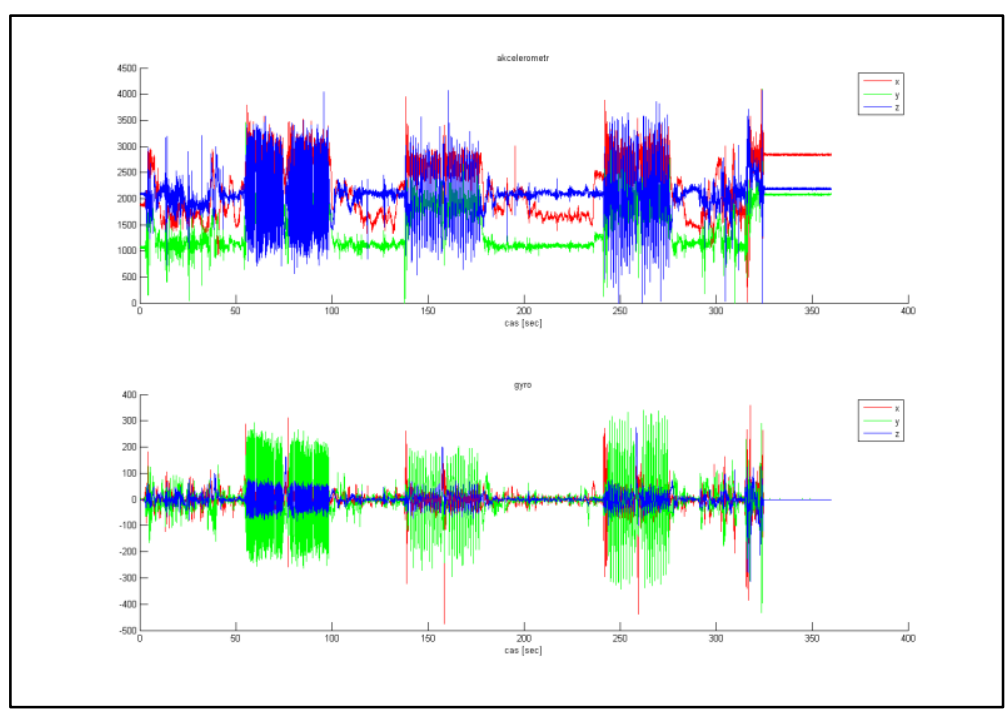

Figure 8. One of the first displays of data set from accelerometer 


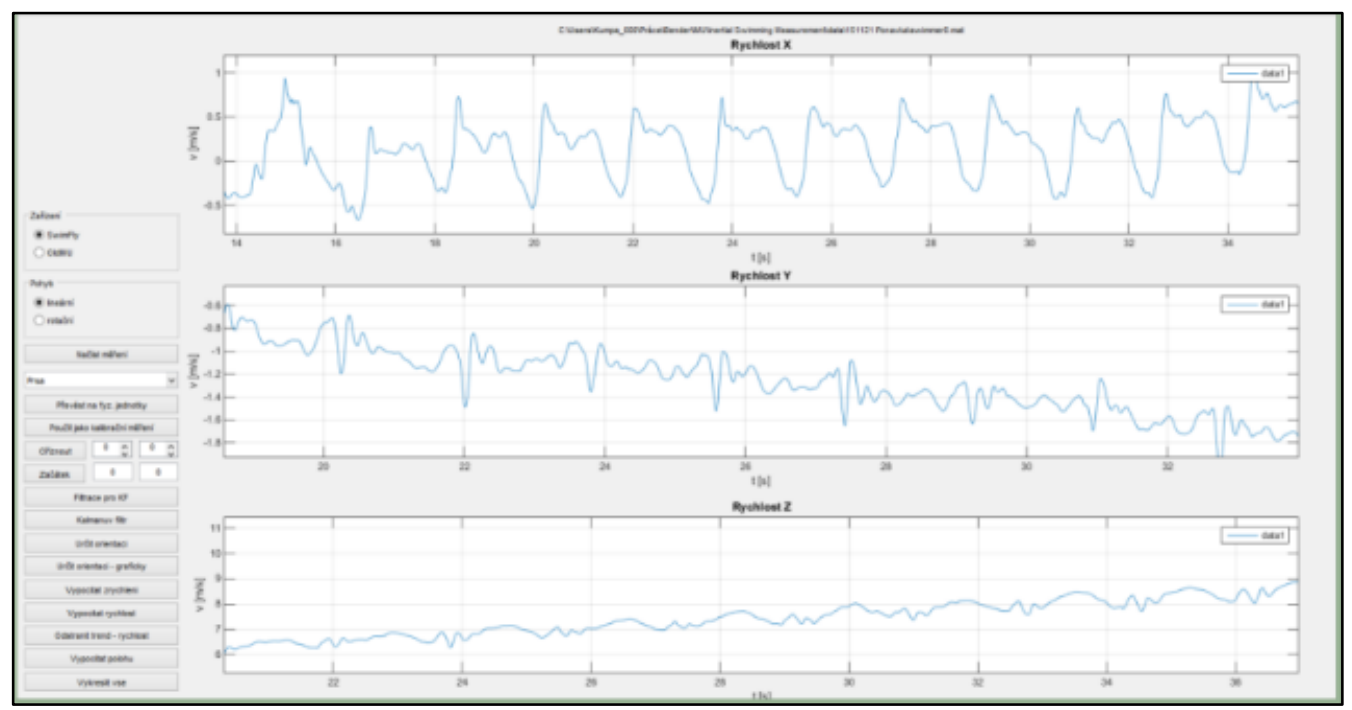

Figure 9. Display of three separate axis of breast stroke speed

Another aim of our development is to synchronize the record from underwater cameras and accelerometers, so that we can (after verifying the validity of the instrument) evaluate mistakes in swimming technique which cause a drop in speed, or on the other hand, what kind of moves result in faster motion of the underwater swimmer.

\section{DISCUSSION}

In our paper, we introduced several methods that are most commonly used for measurement in swimming. Concerning other alternatives, we can mention the measurement in test pools with counter flow, which are however not discussed here, because the measurement conditions in these pools are completely different from normal swimming. They are used for mistakes analysis in swimming technique, e.g. when using video analysis of techniques (Jurák, 2012) and measuring with cameras with SIMI software. Another category of instruments which are used, researched and developed for swimming, are the feedback devices. These are acoustic devices that allow the coach to communicate with the swimmer in the water, the use of robots with an LCD screen on which swimmers can see their technique in real time along with the coach on the bank; the coach can make graphic notes on the display to correct the technique, and other similar devices enabling feedback in real time (Zatoń \& Szczepan, 2013; Hagema, Haeksig, O'Keefe, Stamm, Fickensher, \& Theil, 2013). Given that our aim was to introduce especially those techniques that we ourselves use or plan to include in our research project, it was not possible to introduce many other measuring methods due to limited scope, such as analysis of the power action of the swimmer using a digital dynamometer with a graphical output, which is also marginally solved in our research, and others.

Some of the methods discussed here have the character of theoretical research for analyzing the hydrodynamic effects like the CFD method, which focuses more on the contribution to the theoretical field of hydrodynamic effect of the aquatic environment; other measuring devices are, however, already commercially available (Speedo-meter, Simi Motion cameras and software and others), and are used for the analysis of swimming techniques. Research involves applying them in practice and addressing specific research efforts on swimming techniques. We have brought a comprehensive information about the most recent development engaged in swimming research, which are accelerometers and video systems. A task for further research is verifying the accuracy of measuring devices with accelerometers, which due to their 
sensitive data recorders in all axes are so detailed that for better data display, smoothing of graphic recording is often used, which can cause inaccuracies in the analysis of mistakes in swimming technique. The big advantage of these methods is that they are contactless, and also the low cost of operation and production. However, the question remains whether tachometric methods won't be more accurate or whether the development of research equipment and methods in swimming won't proceed more towards camera systems and appropriate computer software. Thanks to video techniques, swimmers are not limited with anything, there is no need to wear any device or to be tied and preparation for measurement (in the case of permanently installed cameras) is very simple. However, the difficult task is to ensure appropriate recording with sufficient visibility throughout the measurement, which the camera systems require, including transitions of perspectives when shooting under and above water surface.

\section{CONCLUSIONS}

In this paper, we provided information about the development of new measurement methods used for the analysis of swimming techniques in the world, and we described the development and current state of the tachograph measurement method, which is further developed and improved by our team at BUT in Brno, CESA under the leadership of prof. Motyčka. We also brought information about the current situation of the development of our new measuring method of swimmers with accelerometers, which is running test measurements along with the improvement of hardware and software development.

\section{REFERENCES}

1. Barbosa, T., Costa, M., Morais, J., Jesus, S., Silva, A., Batista, J., \& Goncalves, J. (2011). Validation with videometry of an integrated system to assess horizontal intra-cyclic velocity with a mechanical speedo-meter, $\quad 833-835 . \quad$ Retrieved from: https://bibliotecadigital.ipb.pt/bitstream/10198/6117/1/Biomechanics\%20in\%20Sports $\% 2029 \% 20$ $\% 20$ speedo\%20meter\%20validation.pdf

2. Hagema, R., Haeksig, T., O'Keefe, S., Stamm, A., Fickensher, T., \& Theil, D. (2013). Econd generation swimming feedback device using a wearable data processing system based on underwater visible light communication. 6th Asia-Pacific Congress on Sports Technology (APCST).

3. Jurák, D. (2012). Flum. Retrieved from: http://flum.webnode.cz/

4. Lukášek, M. (2000). Uzlové body techniky jako determinanty rychlosti v plaveckém způsobu prsa (Disertační práce). Masarykova univerzita, Brno.

5. Magalhaes, F., Sawaeha, Z., Rocco, D., Cortesi, M., Gatta, G., \& Fantozzi, S. (2013). Effectiveness of an Automatic Tracking Software in Underwater Motion Analysis. Journal of Sports Science and Medicine, 12, 660-667.

6. Motyčka, J. et al. (2001). Teorie plaveckých sportů. Brno: Masarykova Universita.

7. Motyčka, J., Štastný, J., Lepková, H., Pašek, M., \& Bátorová, M. (2013). Kinematic and dynamic analysis of swimming 2009-2013. In: Sport and Quality of Life 2013, 14-23. Brno: Masaryk University.

8. Puel, F., Seifert, L., \& Hellard, P. (2014). Validation of an inertial measurement unit for the determination of the longitudinal speed of a swimmer. Retrieved from: https://www.researchgate.net/publication/279956698_Validation_of_an_inertial_measurement_unit _for_the_determination_of_the_longitudinal_speed_of_a_swimmer

9. Sato, Y., \& Hino, T. (2013). Computational Fluid Dynamics Analysis of Hydrodynamic Force Acting on a Swimmer's Hand in a Swimming Competition. Journal of Sports Science and Medicine, 12(4), 678-689. 
10. Simi Motion 2D/3D - Motion Capture and complex, biomechanical Movement Analysis - syncable, video-based system:. (2015). Retrieved from: http://www.simi.com/en/products/movementanalysis/simi-motion-2d3d.html

11. Stamm, A., James, D., Burkett, B., Hagema, R., \& Theil, D. (2013). Determining maximum push-off velocity in swimming using accelerometers. 6th Asia-Pacific Congress on Sports Technology (APCST).

12. Zatoń, K., \& Szczepan, S. (2013). The Impact of Immediate Verbal Feedback on Swimming Effectiveness. Physical culture and sport, Studies and research. 